\title{
FEMTOSECOND SPECTROSCOPY OF SOLVATED ELECTRON IN AQUEOUS MEDIA
}

\author{
Y. GAUDUEL ${ }^{(1)}$, S. POMMERET and A. ANTONETTI \\ Laboratoire d'Optique Appliquée, CNRS URA 1406, Ecole Polytechnique -ENS Techniques Avancées, \\ F-91120 Palaiseau, France
}

\begin{abstract}
The elucidation of detailed mechanisms of ultrafast events that occur in molecular charge transfer or reaction dynamics has been made possible by recent advances in spectroscopy techniques that use ultrashort laser pulse generation. Ultrashort laser pulses (100 femtoseconds duration, $1 \mathrm{fs}=10^{-15} \mathrm{~s}$ ) allow to initiate selective photochemical processes (single charge transfer for instance) and to obtain unique informations on the dynamics of primary steps of radical reactions involving ultrafast electron or proton transfer: formation of the hydration cage around an electron, encounter pair formation, ion-molecule reaction. Recent investigations on the non-equilibrium states of electron in aqueous media are discussed.
\end{abstract}

\section{Introduction.}

Understanding of solvent influence during charge transfer reactions require to obtain detailed informations on the solvation dynamics of elementary charges and the time-dependent response of solvent molecules to a changing charge distribution. Regarding redox reactions in polar environment, there has been a great deal of discussion over the meaning of frequency factor and the relative role of the collisional and dielectric components solvent friction $/ 1,2$. The exact structure of liquid water is not fully understood at ambient temperature and single charges (electron or derived radicals) or ionic species can be used as microprobe to investigate the local structure and the molecular response of the liquid following a local perturbation (existence of an electric field) $/ 3-5 /$.

The elucidation of detailed mechanisms of ultrafast events that occur during electron or molecule solvation process has been made possible by recent advances in spectroscopy techniques that use ultrashort laser pulse generation $/ 6-8 /$. Ultrashort laser pulses ( 100 femtoseconds duration, $1 \mathrm{fs}=10^{-15} \mathrm{~s}$ ) allow to initiate selective photochemical processes (photoexcitation of molecular probes, photoejection of subexcitation electron) to investigate the role of solvation dynamics during charge transfer. Femtosecond spectroscopy of non-equilibrium states in polar liquids permit to obtain unique informations on the dynamics of primary steps of charge transfer involving single electron or proton: formation of the hydration cage around an electron, encounter pair formation, ion-molecule reaction, electron attachment to solvent molecule, early electron-proton recombination /9-12/.

\footnotetext{
(1) Author to whom correspondence should be addressed.
} 
In polar media (water, alcools...), excess electron implicates intriguing issues on its coupling with solvent molecules since bound states exist only through the interaction with the surrounding medium $/ 3,5,15 /$. It is even more interesting when the liquids presents a high protic character because chemical and physical properties have a determinant influence on the reactivity of single charge (figure 1). Several fundamental points are of interest regarding the coupling between charges (electron, proton) and configurationnal fluctuations of the solvent: role of shallow pre-existing traps; influence of hydrogen bond network; relation between amplitude and spatial frequency of density states for favorable electron or proton localization. In this way, the femtosecond photophysics of water molecules is a powerful tool for the investigation of ultrafast phenomena that occur after an initial energy deposition and charge separation.

Electron Solvation in Protic Polar Media

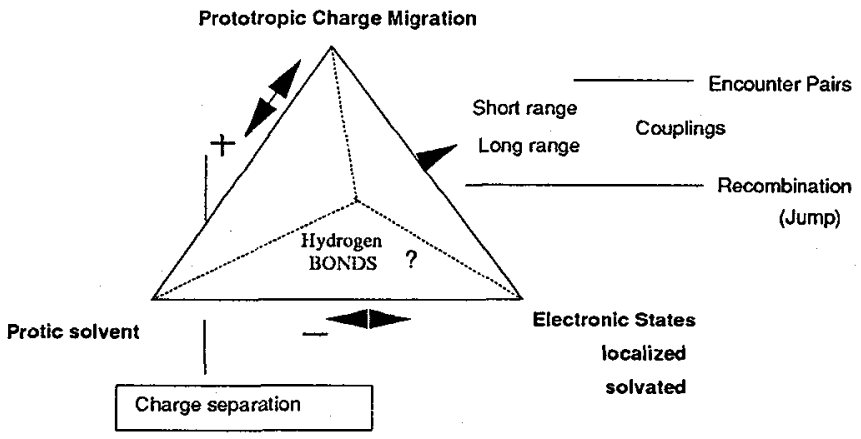

Figure 1: Synthetic representation of primary events involving single charge transfer (electron, proton) in polar protic liquids.

\section{Femtosecond photochemistry in pure liquid water.}

Direct photoionization of water by near UV femtosecond pulse $\left(I>10^{12} \mathrm{~W} \mathrm{~m}^{-2}\right)$ is possible since the light can be absorbed through a nonlinear process. In such experiments, the $8 \mathrm{eV}$ two-photon excitation is above the ionization threshold for liquid water estimated at a very low quantum yield to be around 6.5 $\mathrm{eV}$. The two photon absorption coefficient of pure water at $310 \mathrm{~nm}(4 \mathrm{eV})$ has been estimated to be $410^{-13}$ $\mathrm{mW}^{-1}$ which yields a non-negligible absorption when dealing with multigigawatt peak power pulses $/ 14 /$. During the interaction of ionizing radiation with an aqueous phase, the absorption of energy initiates the ionization process. This step is followed by energy exchanges between excess electron and the molecules of solvent. The energy exchange inside a local region leads to complex couplings between the elementary charge and the protic solvent. The figure 1 represents the primary events linked to the comportment of electronic and prototropic species in polar protic solvent. The initial energy deposition in the bulk phase is followed by the formation of a water cation and transient electronic states (reaction 1):

$$
\mathrm{H}_{2} \mathrm{O}+2 \mathrm{hv} \rightarrow \mathrm{H}_{2} \mathrm{O}^{+}+\left(\mathrm{e}_{\mathrm{q}}^{-}\right)^{?} \rightarrow\left(\mathrm{e}_{\mathrm{th}}\right)^{?} \rightarrow \mathrm{e}_{\text {prehyd }}^{-} \rightarrow \mathrm{e}_{\text {hyd }}^{-}
$$


The investigations on the primary events linked to the hydration of photogenerated electron in aqueous media have been performed using femtosecond laser pulses and absorption spectroscopic methods. In pure liquid water at $294 \mathrm{~K}$, the experimental data obtained at different test wavelenghts in the spectral range $410-1250 \mathrm{~nm}$, have permitted to obtain spectral and dynamical informations on non-equilibrium electronic state configurations $/ 10 /$ and transient prototropic species $\left(11^{a}\right)$.

The analysis of the time-resolved data at different test wavelengths are conducted with kinetical models in which we take into account the convolution of the pump-probe pulses temporal profile and the expected signal rise dynamics including all the different transient species. More precisely the induced absorbance at a specific wavelength $(\lambda)$ and for a time delay $(\tau)$ between the excitation and the probe pulses is the sum of the contribution $A_{i}^{\lambda}(\tau)$ of all the species:

$$
\Delta A^{\lambda}(\tau)=\Sigma A_{i}^{\lambda}(\tau)=\Sigma C_{i} \cdot \varepsilon_{i} \cdot l
$$

In our case of small signal the evolution of the different populations during the pumping and probing follows the relationship:

$$
\Delta A_{i}^{\lambda}(\tau)=\varepsilon_{i}^{\lambda} \cdot l \int_{-\infty}^{+\infty} C_{i}\left(\tau-\tau^{\prime}\right) \cdot C^{\lambda}\left(\tau^{\prime}\right) d \tau^{\prime}
$$

In these expressions, 1 is the interaction length, $C^{\lambda}\left(\tau^{\prime}\right)$ the normalized correlation between the probe and the pump pulse, $\varepsilon^{\lambda}$ and $C_{i}$ respectively the molar extinction coefficient and the concentration of species $i$.

The figures 2 and 3 represent some of the primary events on charge transfer which have been identified in the subpicosecond time scale following the injection of an epithermal electron through two-photon ionization of water molecules. These figures show that ultrafast reactions lead in less than $10^{-12} \mathrm{~s}$ to the formation of primary species: hydronium ion or hydrated proton $\left(\mathrm{H}_{3} \mathrm{O}^{+}\right)$and non-equilibrium configurations of excess electron. Following the photoionization of $\mathrm{H}_{2} \mathrm{O}$ the resulting water cation $\left(\mathrm{H}_{2} \mathrm{O}^{+}\right)$ undergoes, concomitantly to the electron hydration process, an ultrafast charge transfer with one of the surrounding water molecule. The reactivity of this cation is considered as one of the fastest ion-molecule reaction occuring in liquid phase and is believed to take place in less than $10^{-13} \mathrm{~s}$ i.e. the time required for a vibration of a molecule. This ion-molecule reaction leads to the formation of the hydronium ion or hydrated proton $\left(\mathrm{H}_{3} \mathrm{O}^{+}\right)$and the hydroxyl radical $\mathrm{OH}$ : reaction 4 .

$$
\mathrm{H}_{2} \mathrm{O}^{+}+\mathrm{H}_{2} \mathrm{O} \rightarrow \mathrm{H}_{3} \mathrm{O}^{+}+\mathrm{OH}
$$

Femtosecond spectroscopic investigations in the near UV $(410 \mathrm{~nm})$ have permitted to identify an induced absorption, characterized by an uitrashort lifetime and which can be tentatively assigned to the existence of the water cation $\mathrm{H}_{2} \mathrm{O}^{+}$. The relaxation following a monoexponential law would then correspond to the ion-molecule reaction $\mathrm{H}_{2} \mathrm{O}^{+}+\mathrm{H}_{2} \mathrm{O} \rightarrow \mathrm{H}_{3} \mathrm{O}^{+}+\mathrm{OH}$ for which the cleavage rate constant is mesured to be $10^{13} \mathrm{~s}^{-1}$ at $410 \mathrm{~nm}$ (figure 2). We assume that the ultrafast proton transfer from $\mathrm{H}_{2} \mathrm{O}^{+}$to $\mathrm{H}_{2} \mathrm{O}$ is dependent on specific properties of the protic solvent such as the vibrational energy of antisymetric strech. The limiting factor of the cleavage rate constant of the water cation would correspond to the activation energy of a bond cleavage leading to an ultrafast proton transfer from $\mathrm{H}_{2} \mathrm{O}^{+}$to a surrounding water molecule. 

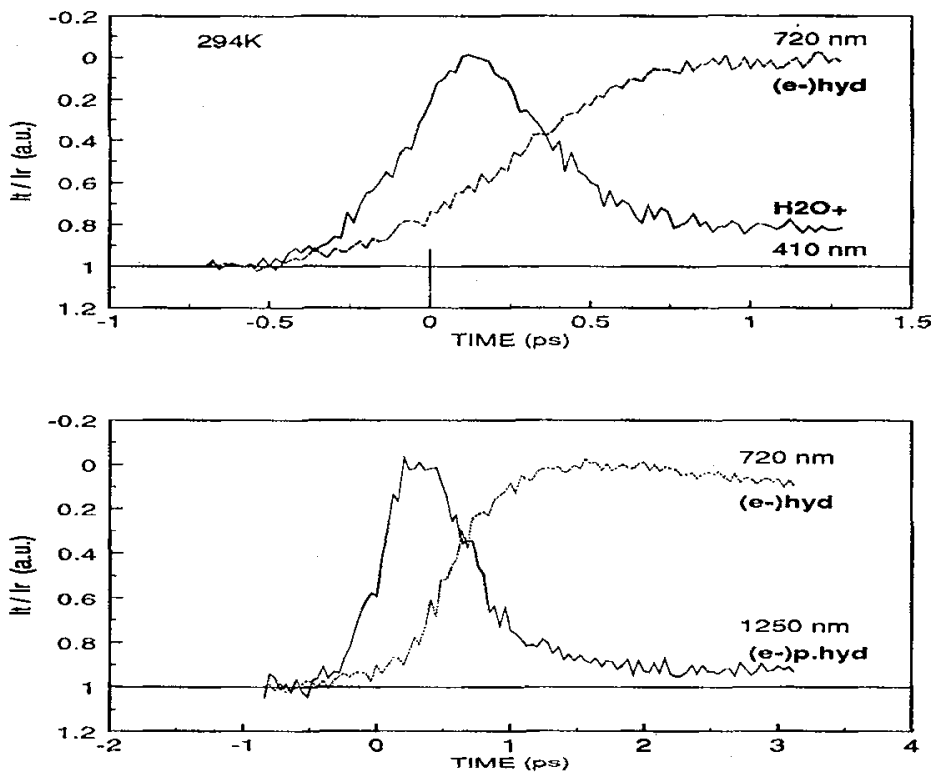

Figure 2: Transient induced absorption obtained at different test wavelengths $(410,720,1250 \mathrm{~nm})$ following photoionization of pure liquid water $(294 \mathrm{~K})$ by ultraviolet femtosecond pulses. $\mathrm{H}_{2} \mathrm{O}^{+}$: water cation, $\left(\mathrm{e}^{-}\right)_{\mathrm{p} . \mathrm{hyd}}$ : pre-hydrated electron, $\left(\mathrm{e}^{-}\right)_{\mathrm{hyd}}$. hydrated electron.

Additional femtosecond experiments exhibit a significant $\mathrm{H} / \mathrm{D}$ isotope effect on the relaxation time of the fastest component $/ 11^{\mathrm{b}} /$. In $\mathrm{H}_{2} \mathrm{O}$ and $\mathrm{D}_{2} \mathrm{O}$ the dry hole $\left(\mathrm{X}_{2} \mathrm{O}^{+}\right)$reacts with an adjoining water molecule and through an ultrafast proton transfer gives the cationic ion $\mathrm{X}_{3} \mathrm{O}^{+}$(hydrated proton) and the radical OX. The H/D isotope effect demonstrates that the ultrafast proton transfer from the water cation to water molecule is likely dependent on specific properties of the protic solvent such as the vibrational energy of antisymetric strech. The limiting factor of the cleavage rate constant of the water cation would correspond to the activation energy of a bond cleavage leading to an ultrafast proton transfer from the cation to a neutral water molecule. Concerning the evolution of the coupling between the water cation and the water molecule during the relaxation process, we should wonder whether this process involves or not some important structural changes including geometrical perturbation of the hydration cage around the water cation. Furtherexperiments are in progress to better understand the mecanism of the fast ion-water molecule reaction in pure liquid water.

Spectroscopic investigations in the visible $(720 \mathrm{~nm})$ and infrared $(1250 \mathrm{~nm})$ have permitted to demonstrate that electron hydration in water proceeds through at least one intermediate state (localized or prehydrated electron) whose the lifetime is in the femtosecond regime (figure 2). This non-equilibrium configuration, characterized by an infrared absorption band extending above $1250 \mathrm{~nm}$, appears in $110 \mathrm{fs}$ and then disappears in $240 \mathrm{fs}$ while a structureless absorption band arises in the visible. The very fast appearance of $\mathrm{e}_{\text {prehyd }}^{-}$is at a time scale short compared to any nuclear motion, solvent dipole orientation or thermal motion of water molecules and its implies that efficient mechanisms involved in the localization process do not require large molecular and dynamical reorganization /10/. Experimentally, the absence 
of a significant continuous shift between the infrared and the visible bands suggests that the relaxation of water molecules in the vicinity of excess electron involves extremely small water motions. In other word an epithermal electron gets to a fully relaxed hydrated state without a dominant dielectric response of the polar medium. At $720 \mathrm{~nm}$, the early decay is attributed to a geminate recombination process $\left(e^{*} . \mathrm{H}_{3} \mathrm{O}^{+}\right.$; $\left.\mathrm{e}^{-} . \mathrm{OH}\right) / 11^{\mathrm{b}} \%$

In heavy water, the hydration process of epithermal electrons having an excess kinetic energy around $1.5 \mathrm{eV}$ occurs through an intermediate state absorbing in the infrared which is attributed to a localized or prehydrated state. The dynamics of electron trapping in pure $\mathrm{D}_{2} \mathrm{O}$ correspond very closely to those in light water. The trapping time ratio between $\mathrm{D}_{2} \mathrm{O}$ and $\mathrm{H}_{2} \mathrm{O}\left(\mathrm{T}_{1(\mathrm{D} 2 \mathrm{O})} / \mathrm{T}_{1(\mathrm{H} 2 \mathrm{O})}\right)$ does not exceed 1.09. This value is lower than i) the ratio of energetic vibrational mode (OH vs OD) which is $\sqrt{2}$ times greater in $\mathrm{H}_{2} \mathrm{O}$ than in $\mathrm{D}_{2} \mathrm{O}$, ii) the estimate of the ratio of the energy loss rate. If the rate of energy loss for the photoejected electron during the thermalization and trapping steps is dependent on the coupling with the most energetic vibrational mode of the OX bond (antisymetric stretch), the time necessary for complete energy dissipation during localization would increase in the same proportion as the ratio of energetic vibrational mode $(\mathrm{OH} / \mathrm{OD})$. As the H/D isotope effect on trapping time is lower than this value $(\sqrt{2})$, it can be concluded that the thermalization step in $\mathrm{D}_{2} \mathrm{O}$ remains very fast and is not the limiting factor in the prehydration process $/ 11^{\mathrm{b}}$.

An important point to draw from femtosecond photochemistry in pure water is that H/D isotope substitution does not affect the relaxation dynamics of prehydrated electrons in neat liquid water. The present data establish that hydration dynamics of subexcitation electron remain largely independent of the physical properties of the polar solvent, such as viscosity, Debye time, vibrational energy of antisymetric strech (OH,OD). Although the Debye relaxation time $\left(\mathrm{T}_{\mathrm{D}}\right)$ is longer in $\mathrm{D}_{2} \mathrm{O}$ than in $\mathrm{H}_{2} \mathrm{O}(10.2 \mathrm{ps}$ vs $8.2 \mathrm{ps}$ at $294 \mathrm{~K}$, there is a discrepancy between the estimate of isotope effect on the hydration time defined by the continuum theory $\left(\mathrm{T}_{\mathrm{L}}=\mathrm{T}_{\mathrm{D}}, \varepsilon_{\circ} / \varepsilon_{o}\right)$ and the experimental values. This discrepancy is in agreement with previous data obtained for light water for which the absence of a continuous shift between the infrared band assigned to a trapped state of electron $\left(\mathrm{e}_{\text {prehyd }}^{-}\right)$and the fully hydrated state $\left(\mathrm{e}_{\text {hyd }}^{-}\right)$supports the existence of a two states process. conclusion that the relaxation of water molecules in the vicinity of an excess electron involves extremely small water motions. The experimental facts lead the conclusion that an epithermal electron reaches its hydration state without a dominant action of the dielectric response of the polar medium and that the relaxation of water molecules in the vicinity of an excess electron would involve small water motions such as librations or $\mathrm{OH}$ vibrations.

Theoretical studies using Molecular Dynamics simulations (MD) and Hole burning suggest that in the two-photon generation of excess electron in pure liquid water, the majority of the excess electrons population will be localized in transient configurations (trapped state or excited hydrated electron). In pure liquid water, the view of the electron solvation as an internal conversion process remains an open question. Theoretical investigations on electron trapping and solvation in pure liquid water are partially consistent with our experimental spectral data and suggest that different populations of non equilibrium electronic configurations (prehydrated states, excited solvated state, trapped electron by pre-existing deep traps) can contribute to the infrared signal before the electron gets its equilibrium state (hydrated state) /16/. More recent simulations consider an internal transition (non adiabatic transition) toward the fully hydrated electron (ground state) /17/.

During the solvation of an excess electron in pure liquid water at ambient temperature, the existence of an isosbestic point in the near IR was, up to now, open to debate $/ 12,18 /$. Very recently, the discremination of a low energy photochemical channel has been obtained in the near infrared spectral region $/ 19 /$. This 
channel favors the formation of a transient encounter pair between the electron and the neoformed prototropic species $\left[\left(\mathrm{H}_{3} \mathrm{O}^{+}: \mathrm{e}: \mathrm{OH}\right)_{\text {hyd }}\right.$ before the total electron hydration process. This non-equilibrium configuration would contribute to the existence of a shoulder in the spectral range $800-900 \mathrm{~nm}$ and demonstrate the absence of an isosbestic point $/ 19 /$. At $820 \mathrm{~nm}$, the computed best fits of the experimental trace assume that the encounter radical pair would occur with an appearance time $\left(\mathrm{T}_{3}\right)$ of $130 \mathrm{t} / \mathrm{-20}$ fs and relaxe following a single transition state (monoexponential law) with a deactivation rate $\left(\mathrm{T}_{4}\right)$ of $330+/-20 \mathrm{fs}$. The contribution of the signal due to this electron-radical pair equals about $30 \%$ at $820 \mathrm{~nm}$. At longer test-wavelength $(880 \mathrm{~nm})$, the early signal decay can be assigned both to a contribution of this encounter pair and to prehydrated electron $\left(\mathrm{e}_{\text {prehyd }}^{-}\right)$. However, at this test wavelength it is not easy to differenciate the exact contributions of the two non-equilibrium electronic states owing to the fact that the different times $\left(\mathrm{T}_{1}, \mathrm{~T}_{2}, \mathrm{~T}_{3}\right.$ and $\left.\mathrm{T}_{4}\right)$ are very close $/ 19 /$. The identification of this non-equilibrium electronic state in the near infrared demonstrates that, at least, two simultaneous electron transfer channels are involved during the photoejecton of epithermal electron from neutral water molcules (figure 3 ). The behavior of the low-threshold ionization process in pure liquid water can lead i) either to a deactivation process by ultrafast reactivity of non fully hydrated electron with the hydronium ion or the hydroxyl radical with geminate proton transfer, ii) or to an internal radiationless transition in the vicinity of prototropic entities to yield fully hydrated electron. In these two cases, the final deactivation process is Ionger than the internal transition of the infrared presolvated electron to the fully hydrated electron.

\section{Electron Solvation in Pure Liquid Water (294K)}

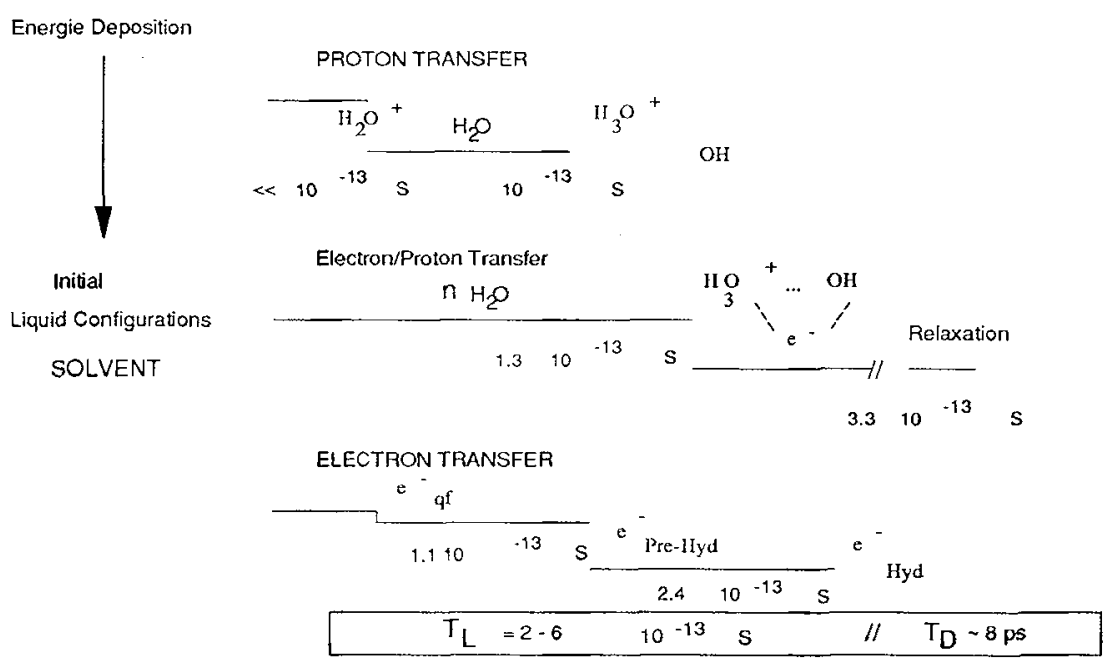

Figure 3: Time-resolved reactional processes occuring in pure liquid water after initial photoejection of excess electron from water molecule by using ultraviolet femtosecond pulses.

Ab initio calculations on the structural characters of water molecules linked to the solvation shells of the hydronium ion in dilute solutions have shown that the radial distribution functions $\mathrm{g}_{\mathrm{OO}}(\mathrm{r})$ and $\mathrm{g}_{\mathrm{OH}}(\mathrm{r})$ exhibit a maximum at $2.48 \AA$ and $3.2 \AA$ respectively $/ 20 \%$. At least two hydration shells can be considered 
over a $7 \AA$ distance range. Consequently, if an electron is directly trapped in the structured hydration shell of $\mathrm{H}_{3} \mathrm{O}^{+}$or $\mathrm{OH}$, the initial charge separation distance would be shorter than the Onsager radius $\left(\mathrm{r}_{\mathrm{c}}=7 \AA\right.$ in water) and very similar to the estimates of reaction radius: $\mathrm{e}^{-} . \mathrm{H}_{3} \mathrm{O}^{+}: 4.5 \AA$, e $\mathrm{e}^{-} . \mathrm{OH}: 6 \AA$. It is important to note that the precursor of the hydronium ion and the hydroxyl radical would relax following an ultrafast proton transfer whose rate constant is faster than the final relaxation step of the trapped electron. This means that a favorable structured environment $\left(\mathrm{H}_{3} \mathrm{O}^{+} \ldots \mathrm{OH}\right)_{\text {hyd }}$ can be created before the electron gets its final equilibrium state. This ion-molecule reaction is in agreement with the hypothesis of an autoionization process of water molecules in which the electron undergoes a coupling with neoformed prototropic species on a short spatial range. The cage effect created by this favorable structure will limite the escape of the excess electron far of the water cation $\left(\mathrm{H}_{2} \mathrm{O}^{+}\right)$. The early reactivity of this hybride pair would involve a fast local reorganization of the hygrogen bond lattice without a significant diffusion process and suggest a pre-eminent role of the ion-molecule reaction to trigger direct electron trapping by siblings. In this hypothesis, if the energy distribution of preexisting traps is determined by solvent fluctuations $/ 21,22 /$ it can be suggested that ultrafast structural solvent reorganization initiated by short lived prototropic species (ion-molecule reaction for instance), would favor the capture of electron by a new local favorable trap state without the electron have to undergo a thermalization process (self trapping induced by "autoionization" of excited states of water molecules). In this hypothesis, the limiting factor for the formation of the favorable structured environment and the subsequent encounter pair formation $\left(\mathrm{H}_{3} \mathrm{O}^{+}: \mathrm{e}^{-} \mathrm{OH}\right)_{\text {hyd }}$ would correspond to the activation energy of the reaction involving ultrafast electron transfer from the water cation to nearest surrounding water molecules. The possibility of a cooperative effect between several water molecules (dimer or molecular cluster) remains open as recently suggested by several authors.

The existence of a low dissociation channel for $\mathrm{H}_{2} \mathrm{O}$ molecules has been suspected to compete with the direct ionization of the $A^{\sim}$ state $\left(1 b_{1} \rightarrow 3 s_{1}\right.$ for instance). Theoretically, the estimate of the energy required to generate electron through vertical ionization (Born-Oppenheimer) is around $8.5 \mathrm{eV}$ within the uncertainty about the value of $V_{0}$ while experimental works have suggested that the threshold energy for the formation of photogenerated hydrated electron through one or two photons will be around $6.5 \mathrm{eV} / 23 /$. In our femtosecond photochemical experiments which use ultraviolet pulse $(E=4 \mathrm{eV})$ and high energy density, the important point to discuss concerns the ionization process which can occur at energy lower than the vertical ionization threshold. The electronic state achieved through a two photon process below the theoretical ionization threshold $(\sim 8.5 \mathrm{eV})$ would correspond to ultrashort excited water molecules (Rydberg states for instance) for which an autoionization process in a condensed phase is possible. Previous studies conclude that a hydrated electron can be obtained when the excitation energy is lower than the energy band gap of water and suggest that an autoionization of water molecules would theoretically be feasable by a thermodynamic process with a free energy $(\Delta G)$ of $5.78 \mathrm{eV} / 24,25 /$. This charge transfer can be considered whether the energy deposition occurs in favorable site geometry for which the thermodynamic process is made possible (low energy photochemical channel). In this way the cooperative effect of two water molecules would enhance photogeneration of electron on short distance i.e. the trapping of the excess electron would occur in the first two solvation shells of the prototropic species. The contribution of such a photochemical channel can explain the kinetic data obtained in the near IR on short or long time scale $/ 18 /$.

Indeed, femtosecond spectroscopic investigations in pure aqueous media provide unique informations on the dynamical coupling between electronic and prototropic species: ultrafast water cation reaction with water molecules $\left(\mathrm{H}_{2} \mathrm{O}^{+}+\mathrm{H}_{2} \mathrm{O}\right)$, relaxation of infrared non-equilibrium states of electrons toward equilibrium configurations, hybride charge transfer leading to encounter pairs (figure 3). Considering this last charge transfer in pure water, it is suggested that structural cooperative effects linked to the presence of prototropic species would favor a charge separation channel equivalent to a low energy photochemical process. In this hypothesis, the trapping of photogenerated electron would occur in the neoformed transient cage for which prototropic species $\left(\mathrm{H}_{3} \mathrm{O}^{+}, \mathrm{OH}\right)$ and few water molecules would be involved. 
3. Computer simulation of the fully relaxed hydrated electron in bulk.

Experimental and theoretical studies of electrons in polar media constitute an active area of research to elucidate the type and location changes induced by a charge separation, the role of the local electro$\mathrm{n}$-solvent interactions and the molecular process associated with the solvent relaxation around an excess electron or prototropic species. Quantum statistical studies using path integral molecular dynamics and Monte carlo statistical methods have permitted to obtain considerable progress on equilibrium structure, optical spectroscopy and relaxation dynamics of hydrated electron $\left(\mathrm{e}_{-{ }_{\text {hyd }}}\right) / 22,26-28 /$.

In this way, we have performed the computation of the fundamental state of an electron in a cavity model composed of six water molecules with an $\mathrm{OH}$ bond orientation toward the centre of the cavity. The methodology used has been described elsewhere and is based on the Splitting Operator Method to resolved the Schrödinger equation $/ 29 /$. The figure 4 represents the interaction of the water molecules of the first. hydration shell with the electron. This interaction is taken into account via a pseudo-potential. The influence of the second solvation shell is treated through the existence of bulk limited to a continuum. The giration radius of the hydrated electron is found to be of the same order of magnitude as computed by statistical methods. For the best value of the cavity radius, more than $60 \%$ of the charge is localized inside the cavity.
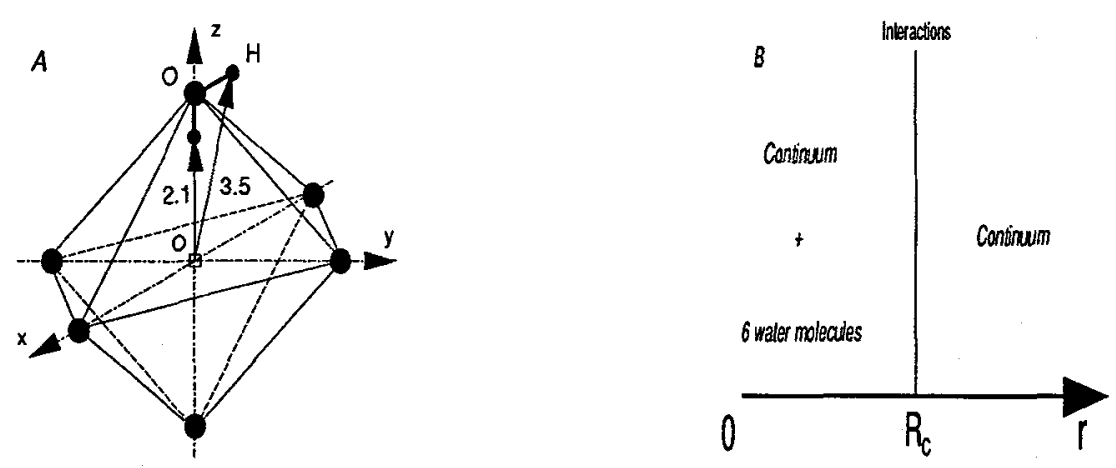

Figure $4:$ : Modelization of the first hydration shell in the present computer simulation. This model is derived from previous work of Kevan, 1981 130/. It considers the presence of six water molecules. $B$ : Representation of the potential distribution used in the present computation; inside the cavity $r \leq R_{c}$ the potential is the sum of the potential due to the 6 water molecules defined in the hydration model and the spherical part of the continuum potential, outside the cavity $r>R_{c}$ the potential is just the continuum potential.

On the Table I we have reported some significant results of our model and have compared them to those obtained by using a complete simulation of the system $\left(\mathrm{nH}_{2} \mathrm{O}+e^{-}\right)$. In this table $\mathrm{K}$ represents the kinetic energy, $\mathrm{P}$ the potential energy, $\mathrm{T}$ the total electronic energy $(T=K+P)$ and $r_{g}$ is the gyration radius of the wavefunction. The present results have been obtained for a cavity size of four Bohr radius. One may not that there exist a good agreement those performed by MD simulations. This fact permitted us to ensure that this model of representation of the coupling between an electron and an aqueous phase is representative of the state of the fully relaxed form of the hydrated electron. 
Table 1 : Comparison between the simulation results obtained by Schnitker and Rossky $131 /$ and those obtained in the present study for a cavity radius of 4 Bohr radius and a temperature of $300 \mathrm{~K}$.

\begin{tabular}{|c|c|c|c|c|}
\hline Parameters & $K(\mathrm{eV})$ & $T(\mathrm{eV})$ & $P(\mathrm{eV})$ & $r_{\mathrm{g}}(\AA)$ \\
\hline References & 2.2 & -2.8 & -5.0 & 2.1 \\
\hline $\begin{array}{c}\text { Schnitker et Rossky (1989) } \\
\begin{array}{c}\text { Present study : } \\
R_{c}=4.0 a_{0} \sim 2.12 \AA\end{array}\end{array}$ & 2.1 & -3.2 & -5.3 & 1.9 \\
\hline
\end{tabular}

\section{Ultrafast charge transfer in concentrated ionic aqueous solutions}

Femtosecond photochemistry of ionic aqueous solutions allow to investigate electron solvation in function of the ionic strength and the nature of ions. Femtosecond photodetachment of electron from a halide $(\mathrm{Cl})$, through charge transfer to solvent spectra (CTTS) can been achieved by pumping with ultraviolet pulses. It has been previously suggested that the femtosecond photolysis channel occurs through a highly excited state of the chloride anion which dissociates to give an epithermal electron and a chlorine atom $/ 32$. Starting from the transient absorption spectra obtained on the picosecond time scale, the primary steps occuring after the femtosecond energy deposition have been shown to be described by the reactions:

$$
\begin{aligned}
& \mathrm{Cl}^{-}+\mathrm{hv} \rightarrow\left(\mathrm{Cl}^{-}\right)^{*} \rightarrow \mathrm{Cl}+\mathrm{e}_{\mathrm{qr}}^{-} \\
& \mathrm{e}_{\mathrm{qf}}^{-}+\mathrm{n}\left(\mathrm{H}_{2} \mathrm{O}\right) \rightarrow \rightarrow \text { hydrated electron }
\end{aligned}
$$

In dilute ionic aqueous solutions $(\mathrm{NaCl}=0.4 \mathrm{M})$, the photodetachment of an electron from $\mathrm{Cl}^{-}$and its subsequent hydration proceed through at least one intermediate step (localization step) absorbing in the infrared and having a lifetime of $250 \mathrm{fs}$. The figure 5 summarizes the influence of the ionic strength on electron trapping and hydration dynamics. The most significant ionic strength effects are observed on the electron hydration dynamics but the dynamics of electron hydration is faster than the formation time $\left(T_{r}\right)$ of the $\mathrm{Na}^{+}, \mathrm{H}^{+}$or $\mathrm{Li}^{+}$atmosphere around $\mathrm{e}_{\text {hyd }}$.

The investigation of early steps following the femtosecond photoionization of halide $\left(\mathrm{Cl}^{-}\right)$in aqueous solution has permitted to demonstrate the existence of specific photochemical channels which are dependent on the nature of the counter ion. It seems reasonable to assume that in concentrated ionic aqueous solution the density and configurational fuctuations provide potential well into which the excess epithermal electron may be directly hydrated. In high concentrated aqueous solutions of hydronium ions, excess electron will be mainly ejected inside the hydration shell of an hydronium ion $\left(\mathrm{H}_{3} \mathrm{O}^{+}\right)_{\mathrm{nH} 2 \mathrm{O}}$ (equation 7). 
(7) $\mathrm{n}(\mathrm{H} 20)+h \vee \rightarrow\left(\mathrm{H}_{3} \mathrm{O}^{+} . . \mathrm{OH}\right)_{\text {hyd }}+\mathrm{e}_{\mathrm{qf}}^{---} \mathrm{k}_{3} \rightarrow\left[\left(\mathrm{H}_{3} \mathrm{O}^{+}: \mathrm{e}^{-}: \mathrm{OH}\right)_{\text {hyd }} \leftrightarrow\left(\mathrm{H}_{3} \mathrm{O} . . \mathrm{OH}\right)^{*}{ }_{\text {hyd }}\right]$

$$
\begin{aligned}
{\left[\left(\mathrm{H}_{3} \mathrm{O}^{+}: \mathrm{e}^{-}: \mathrm{OH}\right)_{\text {hyd }} \leftrightarrow\left(\mathrm{H}_{3} \mathrm{O} . \mathrm{OH}\right)^{*}{ }_{\text {hyd }}\right]-\mathrm{k}_{4} \rightarrow \mathrm{H}+\mathrm{H}_{2} \mathrm{O} } \\
\mathrm{H}_{3} \mathrm{O}^{+} \ldots \mathrm{OH}^{-} \\
\mathrm{e}_{\text {hyd }}^{-}+\left(\mathrm{H}_{3} \mathrm{O}^{+} \ldots \mathrm{OH}\right)
\end{aligned}
$$

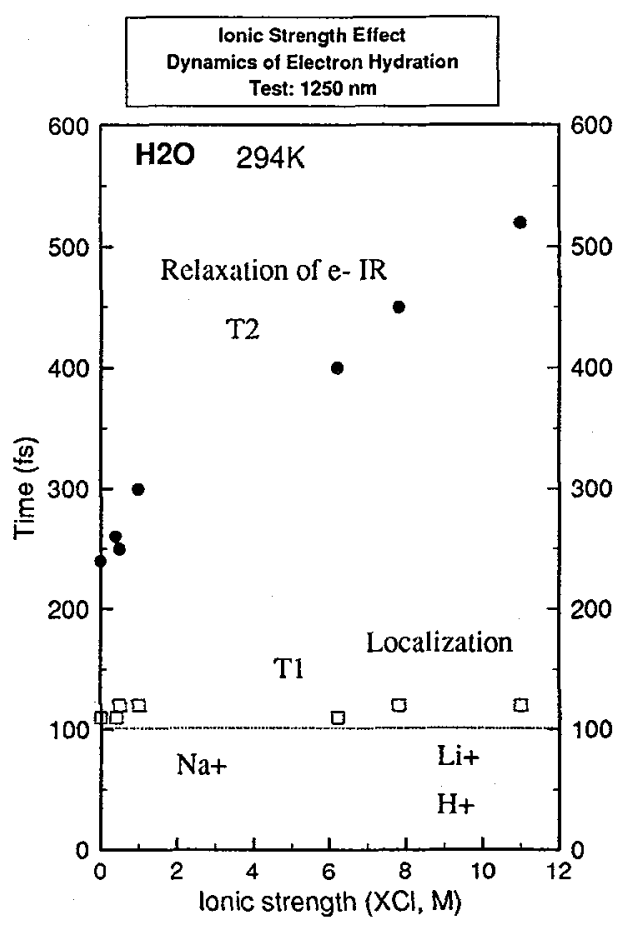

Figure 5: Effect of the ionic stength on the dynamics of electron localization (T1) and hydration (T2) in aqueous solutions.

The fluctuations of structures induced by $\mathrm{H}_{3} \mathrm{O}^{+}$favor the existence of non fully hydrated electron equivalent to a non-equilibrium radical pair. The formation of this pair is found to follow a rate constant of $410^{12} \mathrm{~s}^{-1}$ and has a lifetime of $850 \mathrm{fs} / 33 /$. Indeed, a significant fraction of electron are therefore directly localized in the reaction radius of the hydronium ion i.e. are either present in the hydration shell or tunnel there through a long range. In the evolution of the coupling between the electron-ion pair and water molecules we suggest that the limiting factor of the deactivation dynamics of the encounter pair corresponds to the activation energy of the radical-ion bond cleavage reaction which includes either a proton migration from $\mathrm{H}_{3} \mathrm{O}^{+}$to neighbour water molecules or a local polarization effect on $\mathrm{H}$ bonds.

At this point, let us consider the case of electron comportment in concentrated ionic aqueous solutions for which $\left[\mathrm{H}_{2} \mathrm{O}\right] /[\mathrm{LiCl}]=5$. When the hydration shell around $\mathrm{Li}^{+}$or $\mathrm{Cl}^{-}$correspond to tighly bound water, an important band peaking in the red spectral region can be observed (figure 6 ). The existence of a blue shift is assigned to a change of the electron hydration energy /34/. At the test wavelength corresponding to the maximum of the band $(\sim 660 \mathrm{~nm})$, the dynamics of this ultrafact electron capture equals 
$250 \mathrm{fs}$. In the case of $\mathrm{Li}^{+}$with low electronic affinity, the hydration process would occur through ultrafast electron scattering in deep traps induced by $\mathrm{Li}^{+}$. In this hypothesis, the favorable spatial distribution of deep traps created by the presence of stable counterion $\left(\mathrm{Li}^{+}\right)$would represent a specific order of the liquid for direct electron capture in ground hydrated states. A second channel for electron hydration has been identified in concentrated ionic aqueous solutions. The solvation process involves electron trapping with spectral evidence in the infrared. This non-equilibrium electronic state relaxes toward the fully hydrated state according to a pseudo first-order rate of $1.810^{12} \mathrm{~s}^{-1}$. At this ionic strength, the rotational correlation time $\left(T_{c}\right)$ that is obtained by ${ }^{1} \mathrm{H}$ nuclear magnetic resonance (NMR) is longer than in bulk whereas the ${ }^{1} \mathrm{H}$ NMR relaxation time $T$ is significantly reduced by slow motions of water molecules $/ 34 /$.

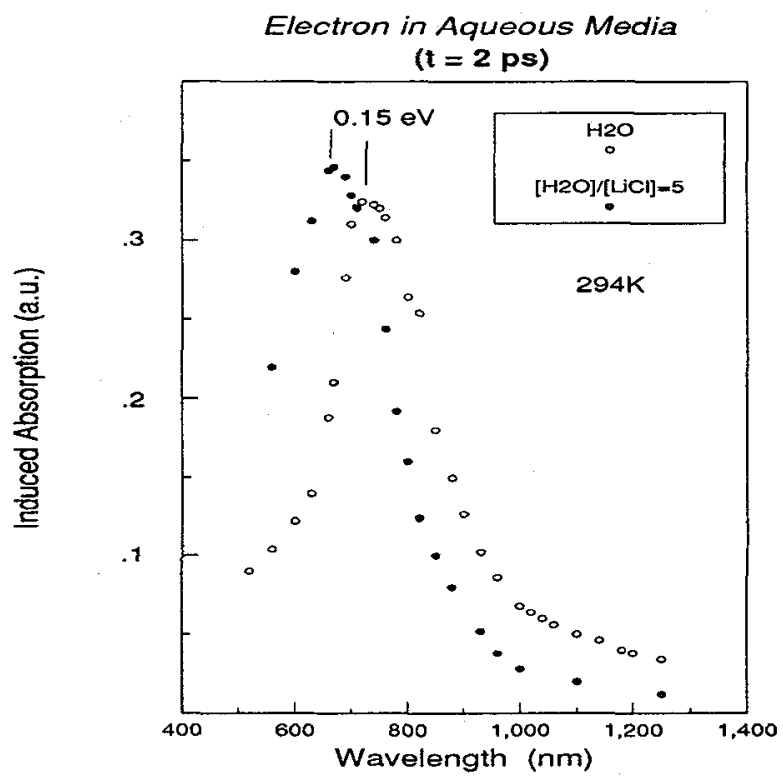

Figure 6: Picosecond spectra of excess electron generated by photodetachment using ultraviolet femtosecond pulses $(E$ excitation $=4 \mathrm{eV}$ ). Two aqueous media are studied: pure liquid water and concentrated ionic aqueous solution ([H2O $/ /[\mathrm{LiCl}]=5)$. A spectral blue shift of $0.15 \mathrm{eV}$ is clearly observed.

The present femtosecond investigations demonstrate that for $\left[\mathrm{H}_{2} \mathrm{O}\right] /[\mathrm{LiCl}]=5$, the lifetime of localized electron is shorter than the formation time (Tr) of the $\mathrm{Li}^{+}$atmosphere around the electron. This implies that in solutions with tighly bound water, the final response of the medium during the hydration involves extremelly small molecular motions. The early behavior decay of the hydrated electron in such concentrated ionic solution has been assigned to the recombination of electron with chlorine atome $\left(e^{-}-\mathrm{Cl}\right)$. This process does not involve trapped electron in the vicinity of lithium ion.

In conclusion, femtosecond photochemistry of pure liquid water and ionic aqueous solutions provide unique informations on the dynamical coupling between electron and prototropic species or other counter ions: ultrafast ion-molecule reaction, identification of several photophysical processes involving infrared non-equilibrium electronic states, characterization of complexe hybride charge transfers leading to encounter pairs. 
Acknowledgments. This work has been supported by grants in aids from Direction des Recherches et

Etudes Techniques (DRET, Paris) and Groupe de Recherches Internationales (Servier, Paris).

\section{References}

/1/ E.W. Castner, G.R. Fleming, B. Bagchi, M. Maroncelli, J. Chem. Phys., 89, 3519 (1988).

/2/ Bagchi, B., Ann. Rev. Chem., (1989), 40, 115 and references therein.

/3/ Hart, E.J., Anbar, M., In "The hydrated electron" Wiley Interseciences, New York, 1970.

14/ N.V. Klassen, "In Radiation Chemistry", Farhataziz and Rodgers Eds., VCH, p 29-64 (1987).

15/ The Chemical Physics of Solvation, Parts A,B,C. In Studies in physical and theoretical chemistry, 38, Edited by R.R. Dogonadze, E. Kalman, A.A. Kornyshev, J. Ulstrup, Elsevier (1988).

16/ Gauduel, Y., Migus, A., Martin, J.L., Antonetti, A., Chem. Phys. Lett., 108, 318 (1984).

77/ Jarzeba, W., Walker, G.C., Johnson, A.E., Kahlow, M.A., Barbara, P.F., J. Phys. Chem., 92, 7039 (1988).

/8/ Y. Gauduel, A. Migus, A. Antonetti "In Chemical reactivity in liquids; fundamental aspects", M. Moreau, P. Turcq Eds., Plenum Press, p15-32 (1988).

19/ Wiesenfeld, J.M., Ippen, E.P., Chem. Phys. Lett., 73, 47 (1980).

/10/Gauduel, Y., Martin, J.L., Migus, A., Antonetti, A. In Ultrafast Phenomena V, Fleming and Siegman Eds, Springer Verlag, New York, (1986) 308. Migus, A., Gauduel Y., Martin, J.L., Antonetti, A., Phys. Rev. Lett., 58, 1559 (1987).

$/ 11 /^{\text {a) }}$ Gauduel, Y., Pommeret, S., Migus, A., Antonetti, A., Chem. Phys. 149, 1 (1990), ; J. Phys. Chem. 93, 335 (1991)

/12/ H. Lu, F.H. Long, Bowman, R.M., K.B. Eisenthal, J. Phys. Chem., 93, 27 (1989); Phys. Rev. Lett., 64, 1469 (1990)

/13/ Baxendale, J.H., Can. J. Chem., 78, 1996 (1977); Kenney-Wallace, G.A., Jonah, C.D., J. Phys. Chem., (1982) 86, 2572; Lewis, M.A., Jonah, C.D., J. Phys. Chem., 90, 5367 (1986); Hirata, Y., Mataga, N., 94, 8503 (1990).

/14/For recent molecular theories, see: Rips, I., Klafter, J., Jortner, J., J. Chem. Phys., 88, 3246, 89, 4288 (1988) and references therein.

/15/ Motakabbir, K.A., Rossky, P.J., Chem. Phys., 1989, 129, 253 and references therein.

/16/ M.C. Messmer, J.D. Simon, J. Phys. Chem., 94, 1220 (1990); R.B. Barnett, U. Landman, A. Nitzan, J. Chem. Phys., 90 (1989) and references therein,.

/17/ Webster, F., Schnitker, J., Friedrichs, M.S., Friesner, R.A., Rossky, P.J., Phys. Rev. Lett., 66, 3172 (1991); Neria, E., Nitzan, A., Barnett, R.N., Landman, U., Phys. Rev. Lett., in press.

/18/ Gauduel, Y., Pommeret, S., Yamada, N., Migus, A., Antonetti, A. In "Ultrafast Phenomena VII", Chemical Physics, 53, Harris, G.B., Ippen, E.P., Mourou, G.A., Zewail, AH Eds, Springer Verlag, 382 (1990).

/19/ Pommeret, S., Antonetti, A., Gauduel, Y., J. Am. Chem. Soc., in press.

/20/ Fornili, S.L., Migliore, M., Palazzo, M.A., Chem. Phys. Lett., 125, 419 (1986).

/21/ Schnitker, J., Rossky, P.J., Kenney-Wallace, P.J., J. Chem. Phys., 1986, 85, 2926.

122/ Romero, C., Jonah, C.D., J. Chem. Phys., 1989, 90, 1877.

/23/Sokolov, V., Stein, G., J. Chem. Phys., 44, 3329 (1966); Nikogosyan, D.N., Oraevsky, A.O., Rupasov, V.I., Chem. Phys., 77, 131 (1983).

24/ Boyle, J.W., Ghormley, J.A., Hochanadel, C.J., Riley, J.F., J. Phys. Chem., 73, 2886 (1969).

125/ Han, P., Bartels, D.M., J. Phys. Chem., 94, 5824 (1990). 
126/ Rossky, P.J., J. Opt. Soc. Am.,B, 7, 1727 (19.90) and references therein.

/27/ Wallqvist, A., Martyna, G., Berne, B.J., J. Phys. Chem., 1988, 92, 1721.

128/ Sprik, M., Klein, M.L., J. Chem. Phys., 1988, 89, 1592.

129/ Pommeret, S., Gauduel, Y., J. Phys. Chem, 95, 4126 (1991)

130/Kevan, L., Radiat. Phys. Chem., 17, 413 (1981)

/31/ Schnitker, J., Rossky, P.J., J. Phys. Chem., 93, 6965 (1989).

132/ Y. Gauduel, S. Pommeret, A. Migus, N. Yamada, A. Antonetti, J. Am. Chem. Soc., 112, 2925 (1990) 133/ Kreitus, I., J. Phys. Chem., 89, 1987 (1985).

134/ Impey, R.M., Madden, P.A., McDonald, I.R., J. Phys. Chem., 87, 5071 (1983). 